\title{
Ligament balancing in TKA: Evaluation of a force-sensing device and the influence of patellar eversion and ligament release
}

\author{
Denis Crottet (1,2), Jens Kowal (1), Sven A. Sarfert (3), Thomas Maeder (4), \\ Hannes Bleuler (2), Lutz-P. Nolte (1), Lutz Dürselen (5) \\ (1) MEM Research Center, University of Bern, Switzerland \\ (2) Laboratoire de Systèmes Robotiques, Ecole Polytechnique Fédérale de Lausanne, Switzerland \\ (3) Department of Orthopaedic Surgery, Inselspital, University of Bern, Switzerland \\ (4) Laboratoire de Production Microtechnique, Ecole Polytechnique Fédérale de Lausanne, Lausanne, Switzerland. \\ (5) Institute for Orthopaedic Research and Biomechanics, University of Ulm, Germany \\ Version of record: Journal of Biomechanics 40 (8), 1709-1715, 2007. \\ http://hdl.handle.net/10.1016/j.jbiomech.2006.08.004
}

\begin{abstract}
Ligament balancing in total knee arthroplasty may have an important influence on joint stability and prosthesis lifetime. In order to provide quantitative information and assistance during ligament balancing, a device that intraoperatively measures knee joint forces and moments was developed. Its performance and surgical advantages were evaluated on six cadaver specimens mounted on a knee joint loading apparatus allowing unconstrained knee motion as well as compression and varus-valgus loading. Four different experiments were performed on each specimen. (1) Knee joints were axially loaded. Comparison between applied and measured compressive forces demonstrated the accuracy and reliability of in situ measurements $(1.8 \mathrm{~N})$. (2) Assessment of knee stability based on condyle contact forces or varus-valgus moments were compared to the current surgical method (difference of varus-valgus loads causing condyle lift-off). The force-based approach was equivalent to the surgical method while the moment-based, which is considered optimal, showed a tendency of lateral imbalance. (3) To estimate the importance of keeping the patella in its anatomical position during imbalance assessment, the effect of patellar eversion on the mediolateral distribution of tibiofemoral contact forces was measured. One fourth of the contact force induced by the patellar load was shifted to the lateral compartment. (4) The effect of minor and major medial collateral ligament releases was biomechanically quantified. On average, the medial contact force was reduced by $20 \%$ and $46 \%$, respectively. Large variation among specimens reflected the difficulty of ligament release and the need for intraoperative force monitoring. This series of experiments thus demonstrated the device's potential to improve ligament balancing and survivorship of total knee arthroplasty.
\end{abstract}

Keywords: Force-sensing device; Ligament balancing; Ligament release; Total knee arthroplasty

\section{Introduction}

Total knee arthroplasty (TKA) has become a routine orthopaedic intervention with high success rate. However, postoperative complications such as joint instability or component loosening can lead to premature wear or failure of the prosthetic joint. Two surgically controllable factors that influence these problems are the tibiofemoral misalignment and the ligament imbalance (Fehring and Valadie, 1994; Moreland, 1988). Misalignment and imbalance can produce a maldistribution of the tibiofemoral contact forces which generates an overload of one compartment, thus accelerating the wear process. With the recent introduction of surgical navigation systems, accuracy of three-dimensional tibiofemoral alignment can be improved (Decking et al., 2005; Haaker et al., 2005; Stulberg, 2003; Jenny and Boeri, 2001). Nevertheless, the ligament tensions remain only qualitatively assessed with manual trial movements or with basic mechanical tools. In order to improve the ligament balancing procedure, a force-sensing device for intraoperative measurement of the condyle contact forces was developed.

The force-sensing device, which was described in detail in a previous publication (Crottet et al., 2005), consists of two sensing plates, one for each condyle, a tibial base plate, which is fixed by pins, and a set of lateral and medial spacers, which allow the device thickness to be adapted to the patient-specific tibiofemoral gap. The entire device fits, after the initial tibial cut, inside the knee joint with the patella in its 
anatomical position. The correction of tibiofemoral alignment and knee stability can thus be planned simultaneously at the beginning of the surgery when all the correction means, such as bone cuts, are still at the surgeon's disposal. Performing the assessment of the joint stability at an early stage of the TKA procedure can offer the possibility to plan patient-specific femoral component rotation, to minimize bone resection, to facilitate the preservation of joint line and finally to minimize the final correction with ligament releases. Each sensing plate of the device contains three deformable bridges which allow determining the amplitude and location of the load applied with respective intrinsic accuracies of $1.4 \mathrm{~N}$ and $0.6 \mathrm{~mm}$ (Crottet et al., 2005). With these measurements, the net varus-valgus moment, which is considered as the biomechanical parameter characterizing the ligament imbalance, can be computed.

A few measurement systems aimed at providing a quantitative measurement of the ligament imbalance have been reported in the literature (Winemaker, 2002; Morris et al., 2001; Takahashi et al., 1997; Wallace et al., 1998; Attfield et al., 1994). However, they show various drawbacks such as a low accuracy, no realtime measurement, the necessity of a patellar eversion that may significantly modify the mediolateral force distribution or a stability assessment at the end of the surgery when the possibilities of correction are limited. It is nevertheless interesting to note that each of these devices measures a different biomechanical parameter: Winemaker (2002) measures extension gaps, Morris et al. (2001) compressive forces, Takahashi et al. (1997) and Wallace et al. (1998) contact pressure and finally Attfield et al. (1994) angular deviations of a scale-like device. This indicates that there is no standard interpretation in biomechanical terms of what the surgeon intuitively calls ligament balancing.

One means to correct ligament imbalance is the ligament release. One standard approach consists of a partial resection of the lateral or medial collateral ligament close to the bone, at its attachment point. By cutting a part of the fibers constituting the ligament, its stiffness and thereby its tension decreases. Even though ligament release is commonly used to correct imbalance, its biomechanical effect in terms of knee joint forces is poorly documented.

The goal of the present study, focused on surgical management of ligament balancing, was therefore to evaluate the in situ functioning and clinical advantages of the novel force-sensing device through (a) identifying the biomechanical parameter that the surgeon intraoperatively assesses during ligament balancing and (b) quantifying the effect of patellar eversion and collateral ligament release in terms of tibiofemoral contact forces.

\section{Methods}

The force-sensing device was tested in six human cadaver knees from donors with an average age of 82 years (63-90). The surgical preparation of each specimen consisted of the two first steps of TKA, i.e. accessing the joint with a medial parapatellar approach followed by the resection of the tibial plateau. The force-sensing device was then introduced and the specimen mounted on a knee joint loading apparatus (Fig. 1) previously described by Dürselen et al. (1995). This machine provides all the degrees of freedom needed for unconstrained knee motion and allows the monitoring of the relative angular position between the femur and tibia. Compression loads and varus-valgus moments can be applied by using a system of weights and pulleys. Additionally, quadriceps forces can be simulated by loading the patellar tendon with weights.

To validate the in situ measurement of forces, the knee joint was loaded in full extension with axial compressive weights ranging from 1 to $15 \mathrm{~kg}$. This range was selected on the basis of a first indicative in vitro experiment (Crottet et al., 2005) in which contact forces of each tibial compartment ranged from 40 to $70 \mathrm{~N}$ when the knee joint was in full extension and mechanically unconstrained. The sum of the medial and lateral contact forces measured by the device, i.e. the total force transmitted through the tibiofemoral joint, was then compared to the applied loads.

The second part of the study aimed at identifying the biomechanical parameter that the surgeon currently assesses during ligament balancing. Intraoperatively, the surgeon manually compares the varus and valgus forces that must be applied at the ankle to create a medial or lateral condyle lift-off. With a simplified twodimensional model (Fig. 2), it can be theoretically demonstrated that this corresponds to a balancing of the medial and lateral condyle contact forces. The model considers the five major forces involved in the varusvalgus stability: the medial and lateral collateral ligament forces (FML, FLL), the medial and lateral contact 
forces (FMC, FLC) and an external varus force (FEX). The centre of rotation, which in Fig. 2 corresponds to the contact point of the medial condyle, is taken as the reference for the computation of moments. In this model, bones are considered as rigid bodies and ligaments as springs.

The equilibrium condition for the moments yields

$$
X_{\mathrm{ML}} \cdot F_{\mathrm{ML}}-X_{\mathrm{LL}} \cdot F_{\mathrm{LL}}+X_{\mathrm{C}} \cdot F_{\mathrm{LC}}+Y \cdot F_{\mathrm{EX}}=0
$$

The resultant ligament moment MLIG can be defined as

$$
M_{\mathrm{LIG}}=X_{\mathrm{ML}} \cdot F_{\mathrm{ML}}-X_{\mathrm{LL}} \cdot F_{\mathrm{LL}}
$$

This moment and the contact forces are clearly influenced by the external force $F_{\mathrm{EX}}$, which can be seen as the variable of the system. Due to the hypothesis of rigid bodies and springs, this influence can be reasonably expressed by linear relationships, with $C, D$ being the proportionality factors

$$
\begin{aligned}
& M_{\mathrm{LIG}}\left(F_{\mathrm{EX}}\right)=M_{\mathrm{LIG}}(0)+C \cdot F_{\mathrm{EX}} \\
& F_{\mathrm{LC}}\left(F_{\mathrm{EX}}\right)=F_{\mathrm{LC}}(0)+\mathrm{D} \cdot F_{\mathrm{EX}}
\end{aligned}
$$

The combination of Eqs. (1), (2), (3) and (4) can then be written as

$$
M_{\mathrm{LIG}}(0)+C \cdot F_{\mathrm{EX}}+X_{\mathrm{C}} \cdot F_{\mathrm{LC}}(0)+X_{\mathrm{C}} \cdot D \cdot F_{\mathrm{EX}}+Y \cdot F_{\mathrm{EX}}=0
$$

when there is no external force applied $\left(F_{\mathrm{EX}}=0\right)$, Eq. (5) gives

$$
M_{\mathrm{LIG}}(0)+X_{\mathrm{C}} \cdot F_{\mathrm{LC}}(0)=0
$$

where $F_{\mathrm{LC}}(0)$ corresponds to the lateral contact force with no external load. When there is a varus load $\left(F_{\mathrm{EX}}=F_{\text {varus }}\right)$ that generates a lateral condyle lift-off $\left(F_{\mathrm{LC}}\left(F_{\text {varus }}\right)=0\right)$, Eq. (5) gives

$$
M_{\mathrm{LIG}}(0)+C \cdot F_{\text {varus }}+Y \cdot F_{\text {varus }}=0
$$

The relationship between the varus load required for a condyle lift-off and the initial lateral contact force can be deduced from Eqs. (6) and (7)

$$
F_{\text {varus }}=X_{\mathrm{C}} /(C+Y) \cdot F_{\mathrm{LC}}(0)
$$

The same approach can be adopted for the case of a valgus external force and a medial condyle lift-off

$$
F_{\text {valgus }}=X_{\mathrm{C}} /(C+Y) \cdot F_{\mathrm{MC}}(0)
$$

With Eqs. (8) and (9) it can finally be concluded that the relative difference of the forces required for a condyle lift-off is equal to the relative difference of the contact forces:

$$
\text { Relative imbalance }=\left(F_{\text {varus }}-F_{\text {valgus }}\right) /\left(F_{\text {varus }}+F_{\text {valgus }}\right)=\left[F_{\mathrm{LC}}(0)-F_{\mathrm{MC}}(0)\right] /\left[F_{\mathrm{LC}}(0)+F_{\mathrm{MC}}(0)\right]
$$

In order to experimentally validate this equivalence as well as to compare this approach to the device's measurements, the relative imbalance was assessed on the basis of three different parameters: (a) the weights required for condyle lift-off, which corresponds to the current surgical approach described above, (b) the contact forces with no external load and (c) the varus-valgus moments with no external load. The knee joint was loaded with varus and valgus forces with weights increasing from 0 to $1 \mathrm{~kg}$. If the condyle lift-off was not achieved before $1 \mathrm{~kg}$, larger weights were used. Since during the surgery the ligament imbalance is assessed in full extension and in $90^{\circ}$ flexion, the experiment was performed in both positions. For each weight, the contact forces and the varus-valgus moments were measured with the device. The relative imbalance of each method, i.e. the ratio between the difference and the sum of the weights, forces or moments, was computed. By definition, a positive/negative value was related to a lateral/medial imbalance. The statistical difference of the three approaches was evaluated with a Friedman ANOVA test followed by individual post hoc comparisons with Dunnett's test, where the weight- based approach was taken as the control group. 


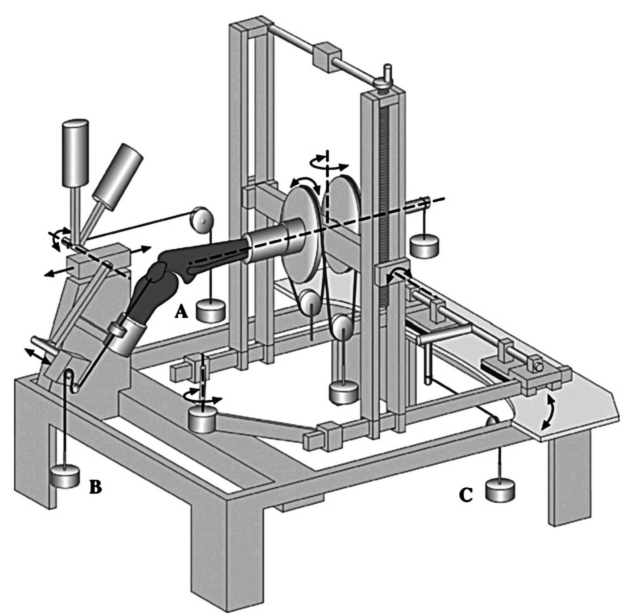

Fig. 1. The knee joint loading apparatus used for the validation experiments. The arrows indicate the degrees of freedom of the machine, which allows unconstrained knee motion. Compression loading (a), quadriceps force simulation (b) or varus-valgus loading (c) can be carried out with a system of weights and pulleys.

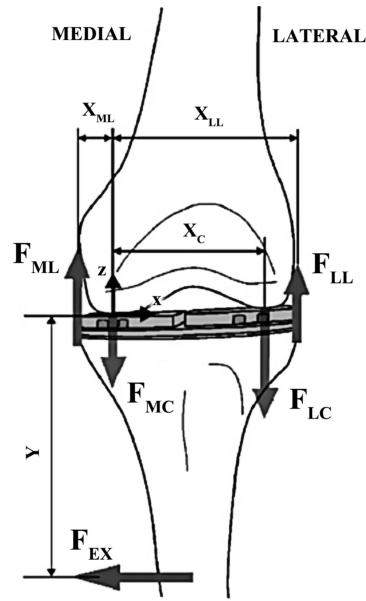

Fig. 2. A simplified two-dimensional biomechanical model of a knee joint experiencing varus or valgus load to create a lateral or medial condyle lift-off. The five major forces involved in the varus-valgus stability, which are the collateral ligament forces $\left(F_{\mathrm{ML}} ; F_{\mathrm{LL}}\right)$, the contact forces $\left(F_{\mathrm{MC}} ; F_{\mathrm{LC}}\right)$ and the external force $\left(F_{\mathrm{EX}}\right)$, are taken into account.

Since the possibility of having the patella in its anatomical position during the measurement is claimed to be a major advantage, the effect of a patellar eversion on the mediolateral contact force distribution was evaluated. To simulate patellar load induced intraoperatively by the passive tension of the quadriceps, the patellar tendon was loaded with weights ranging from 1 to $5 \mathrm{~kg}$. The medial and lateral contact forces perpendicular to the tibial plateau were measured with the device. In order to evaluate the mediolateral distribution of the contact force induced by the patellar load, the variation, i.e. the slope, of the medial and lateral contact forces as a function of the applied weights were compared. Comparing the variation and not the absolute amplitude of the contact forces allows isolating the contribution of the patella from the constant contribution of other soft-tissue structures, such as collateral ligaments. The data were normalized so that the sum of the relative medially and laterally transferred loads corresponds to $100 \%$. These measurements were performed at different flexion angles $\left(0^{\circ}, 30^{\circ}, 60^{\circ}\right.$ and $\left.90^{\circ}\right)$ and with or without a patellar eversion. The statistical significance of the influence of patellar eversion and flexion angle on the mediolateral distribution of the contact force induced by the patellar load was determined with a factorial ANOVA test.

In a final step, the biomechanical effect of medial collateral ligament releases was quantitatively evaluated. With interarticular spacers applying stress to the ligaments, a minor followed by a major medial collateral ligament release was performed with the knee in full extension. A minor or major release corresponded, respectively, to the resection of one third or two thirds of the ligament-bone attachment. The spacer size was selected to have an initial medial contact force comprised between 50 and $100 \mathrm{~N}$ without any additional load. The medial and lateral contact forces were recorded after each surgical action and compared to the initial value. In order to quantify the effect of the ligament release on the tibiofemoral alignment, the variation of the varus-valgus angle resulting from the releases was measured as well. Finally, the initial and ultimate contact forces were measured at $0^{\circ}, 30^{\circ}, 60^{\circ}$ and $90^{\circ}$ flexion to evaluate the effect of ligament releases throughout the knee flexion. The influence of the flexion angle was determined with a one-way ANOVA test.

\section{Results}

All the curves obtained during the compression loading experiment were highly linear $\left(r^{2}=1.00\right)$ and the measured slopes ranged between 0.96 and 1.03, thus being very close to the ideal value of 1 (Fig. 3). Over all the measurements, the average absolute difference between the applied and measured compressive force was $1.8 \mathrm{~N}$ with a standard deviation of $1.7 \mathrm{~N}$ and the average relative difference was $3 \%$ with a standard deviation of $2 \%$. 
A typical curve obtained during a varus-valgus loading in full extension (Fig. 4) presents three distinct areas: (1) the lateral condyle is in contact; (2) both condyles are in contact; (3) the medial condyle is in contact. In each area, a linear relationship between the applied and measured varus-valgus moments was found, thus being in agreement with the hypothesis of Eq. (3). The slope was lower in the situation where only one condyle was in contact than where both were. The Friedman ANOVA test demonstrated that the three approaches to assess the knee stability (measurement of the weights required for condyle lift-off, measurement of the contact forces or measurement of the varus-valgus moments with no external load) are not all equivalent $(p=0.017)$. Post hoc analysis showed that the moment-based approach is significantly different from the weight-based $(p=0.005)$ while the force-based is not $(p=0.417)$. Taking the current surgical approach, i.e. the weight-based assessment, as a reference (Fig. 5), the force- based assessment has an average difference of $0 \%$, thus demonstrating the expected equivalence of the two approaches. However, the moment-based assessment showed a tendency for lateral imbalance. Even though the average difference was small (6\%), large differences were found in some cases, such as in the third knee. Similar results were obtained for the measurements performed in $90^{\circ}$ flexion but with an average difference of imbalance of $4 \%$ and $7 \%$, respectively, for the force-based and moment-based assessments.

In the patellar load distribution experiment, the statistical analysis showed that the flexion angle was not a significant factor $(p=0.212)$. Therefore, the medially and laterally transferred loads were averaged over the different knee positions. The mediolateral distribution of the contact force induced by the patellar load was significantly different $(p<0.001)$ with the patella everted from standard anatomical position: one fourth of the patellar load was shifted from the medial to the lateral side with a patellar eversion (Fig. 6). Additionally, it can be noted that the patella in its anatomical position already gave a non-symmetric mediolateral distribution of the contact forces since the average laterally transferred load was $62 \%$ without a patellar eversion.

Finally, the minor and major medial ligament releases reduced the medial contact force in full extension by $20 \%$ and $46 \%$ on average (Fig. 7). The large standard deviations, $19 \%$ and $25 \%$, demonstrated the high variability of the biomechanical effect of a ligament release among the specimens. The lateral contact force was just slightly influenced by the medial ligament releases (Fig. 8), since an average increase of $9 \%$, which is only one fifth of the reduction on the medial side, was measured. For all specimens, the variation of the varus-valgus angle was below $0.2^{\circ}$. This indicates that the ligament release did not substantially modify the tibiofemoral mechanical axis. Finally, the effect of ligament releases seems to vary with knee flexion, being apparently less relevant at $30^{\circ}$ and $60^{\circ}$ than in full extension or $90^{\circ}$ flexion (Fig. 9), although statistical significance was not achieved $(p=0.170)$.

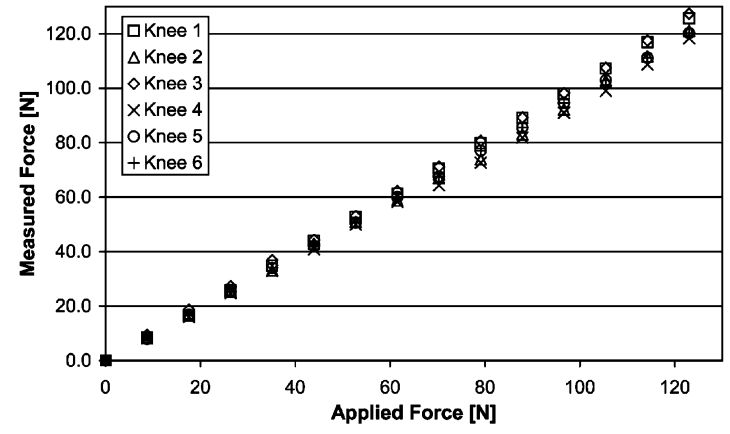

Fig. 3. Comparison between the measured and applied forces during the compression loading experiment. The linearity and the small deviation of the observed slopes from the ideal value of 1 demonstrate the accuracy and reliability of the in situ force measurement.

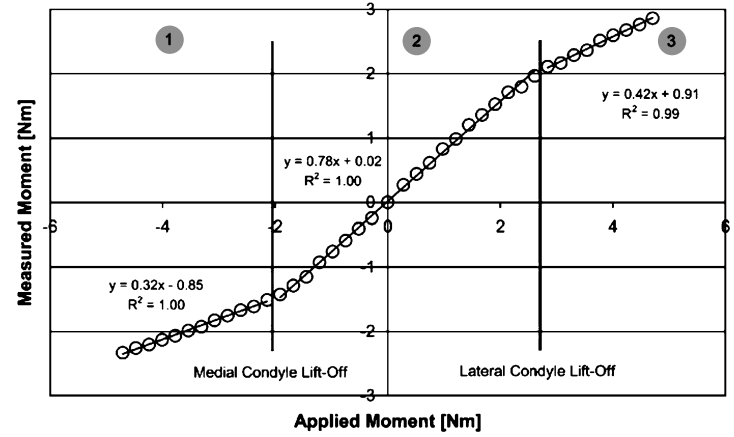

Fig. 4. Typical comparison for one specimen between the applied and measured moments during varus-valgus loading in full extension. Three different situations can be distinguished: (1) the lateral condyle is in contact; (2) both condyles are in contact; (3) the medial condyle is in contact. Each area presents a specific linear relationship and the difference of the slopes expresses the different contribution of the collateral ligaments in the situations where only one or both condyles are in contact. 


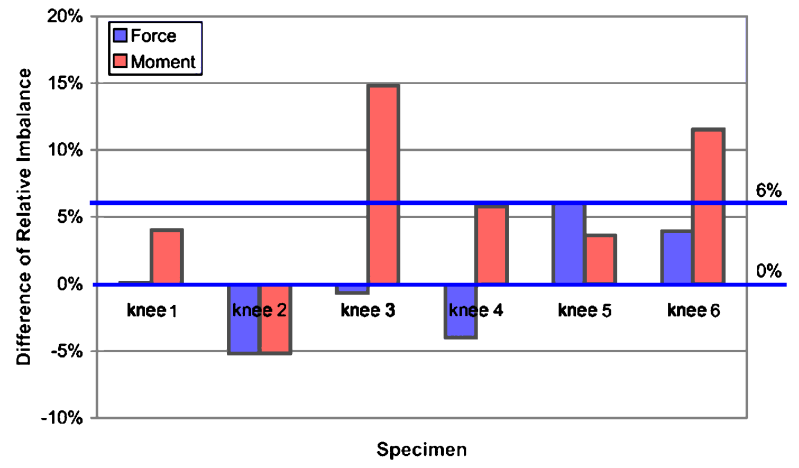

Fig. 5. Assessment of the relative ligament imbalance in full extension based on different approaches. The weight-based approach, which corresponds to the current surgical approach, is taken as a reference. On average, the forcebased approach was equivalent $(0 \%)$ while the momentbased approach showed a slight lateral imbalance $(6 \%)$.

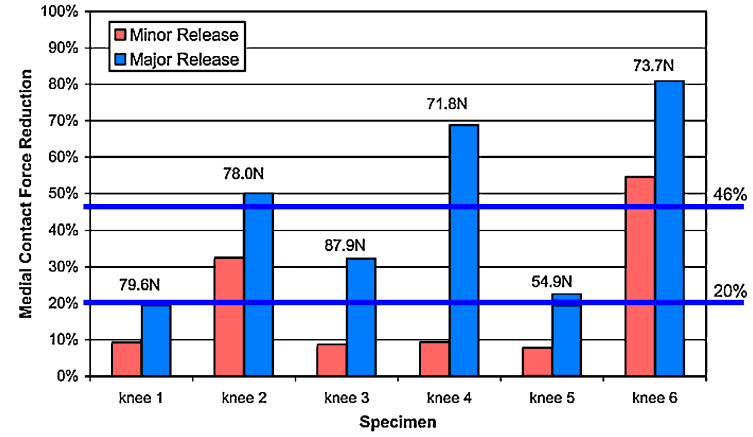

Fig. 7. Relative reduction of the medial contact forces following a minor and a major medial collateral ligament release in full extension. The grey and black horizontal lines show the average force reduction due to a minor and a major release, respectively. The values above bars indicate the initial force amplitude.

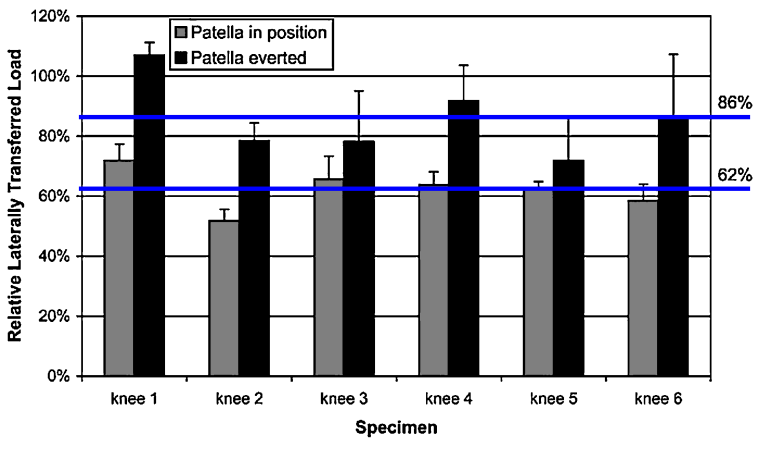

Fig. 6. Relative laterally transferred loads during the patellar load distribution experiment, showing that the patellar eversion significantly affected the mediolateral distribution of the contact forces. One fourth of the patellar load was shifted from the medial to the lateral side. The grey and black horizontal lines correspond to the average loads with the patella in position or everted respectively.

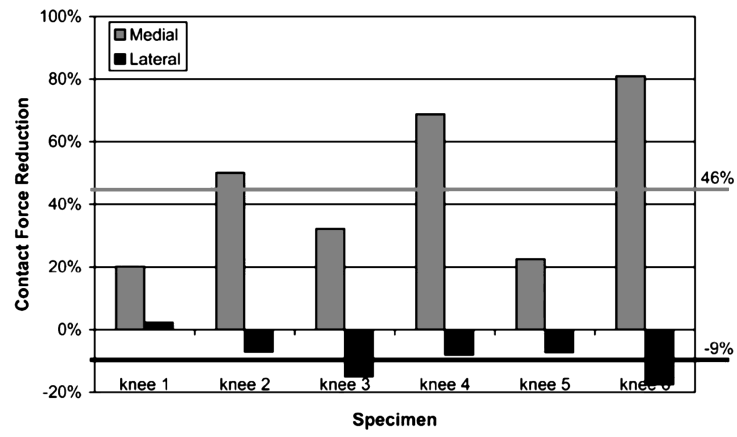

Fig. 8. Relative reduction of the medial and lateral contact forces following a major medial collateral ligament release in full extension. With an average increase $(9 \%)$ equivalent to a fifth of the medial force reduction $(46 \%)$, the influence of a medial ligament release on the lateral contact force was minor.

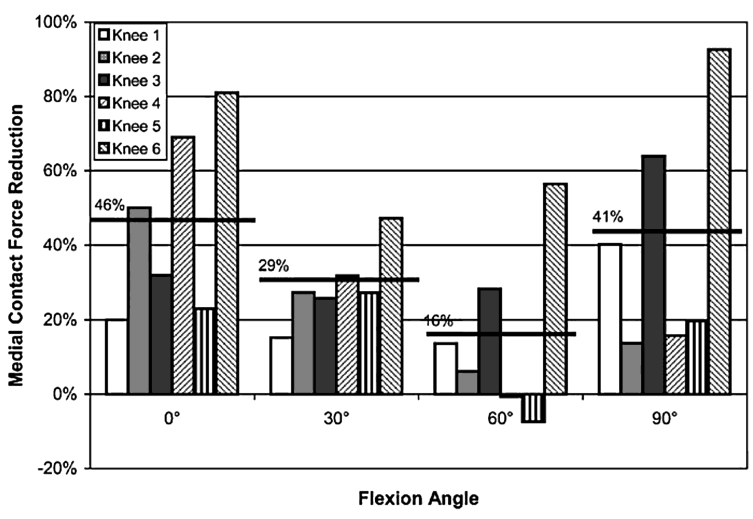

Fig. 9. Variation of the effect of a major medial collateral ligament release along knee flexion. The release was less effective at $30^{\circ}$ and $60^{\circ}$ flexion than in full extension or $90^{\circ}$ flexion. The black horizontal lines correspond to the average values at the different positions.

\section{Discussion}

Since a well-balanced distribution of medial and lateral contact forces avoids overload of one compartment and thus premature wear, knee imbalance assessment is a key point in TKA. In order to provide intraoperative assistance for this assessment, a force-sensing device was developed. Its performance and clinical advantages were evaluated with six cadaver knees and four different experiments: compression loading to validate the device measurements, varus-valgus loading to compare the device's measurements to the current surgical approach, patella loading to demonstrate the advantage of keeping the patella in its anatomical position during the measurement and finally ligament releasing to quantify its biomechanical effect in terms of forces. 
The very good agreement between applied and measured tibiofemoral contact forces during the compression loading experiment proved the accuracy and reliability of the in situ device measurements.

The shape of the curves obtained during the varus-valgus loading experiment can be biomechanically explained. When the joint opens after condyle lift-off, the lateral or medial collateral ligament bears a relevant part of the applied load. Since this part is not measured by the device, it therefore appears as a drop in the slope. The difference of the slopes between the areas 1 and 3 also demonstrates that the lateral and medial ligaments can have different stiffnesses. The determination of the weight causing a medial or lateral condyle lift-off allowed the comparison of the current surgical approach to assess the knee instability to the approach based on the measurement of the contact forces or varus-valgus moments. Overall, the three methods gave consistent results. Weight-based imbalance assessment was equivalent to force-based, as expected by the simplified two-dimensional model. Moment-based imbalance assessment, which is assumed to be the optimal approach (Crottet et al., 2005), showed a tendency of lateral imbalance. This can be anatomically explained by the fact that the medial condyle is smaller than the lateral and consequently has its contact point closer to the centre of the joint. It should also be noted that this cadaver model does not take into account any arthritic deformities, which knees selected for TKA would have. Bone deformities can significantly shift the condyle contact points and thereby further increase the difference between the force-based and moment-based imbalance assessment. The clinical advantage of a moment-based approach is the assurance of equal tibial contact pressure once the symmetric femoral component is in place. With a force-based approach, the difference of lever arms before and after the component placement alters the final force distribution according to the principle of mechanics equilibrium.

The advantage of having the patella in its anatomical position during the measurement was shown by the significant influence of the patellar eversion on the mediolateral distribution of the condyle contact forces. One fourth of the contact force induced by the patellar load was shifted to the lateral compartment with a patellar eversion. Although the influence of patellar eversion on the mediolateral force distribution is intuitive, it is the first time, to the authors' knowledge, that the effect is quantified in terms of forces. Only one recent study (Lüring et al., 2005) compared the influence of the patellar eversion and subluxation to the physiological situation in terms of angular variation. With a given interarticular load of $150 \mathrm{~N}$ for the medial and lateral side, the patellar eversion increased the leg axis by $2.9^{\circ}$ valgus. Although a quantitative comparison to the present study is not possible, the measured effects are qualitatively consistent. Therefore, the clinical practice of everting the patella biased the stability assessment and implies an imbalance towards the medial compartment when the patella is brought back in its original place. Finally, the fact that the contact force induced by the patellar load was unevenly distributed with the patella in its anatomical position could be due to a non-symmetrical contact area between the patella and the femoral trochlea, to a valgus knee deformation or to the medial incision of the knee joint.

Since the experimental set-up allowed all the parameters to be kept constant during the ligament release, the change in total joint force can be supposed equal to the change in ligament tension. On average, the minor and major medial collateral ligament releases reduced the medial contact force $(20 \%$ and $46 \%$, respectively). The large variation among the specimens illustrates the difficulty of ligament release, which is twofold: (a) the difficulty to ensure reproducible resection and (b) the difficulty to evaluate the release effect in terms of forces, which depends on the ligament stiffness of the specimen. An intraoperative force monitoring during this delicate surgical action could provide further support to ensure an accurate, patientspecific adjustment of the ligament tensions. The increase of the lateral force after the major medial ligament release was only a fifth of the decrease on the medial side. Consequently, in the case of a small ligament imbalance requiring only a minor force reduction, the effect of the ligament release on the opposite side of the surgical action can be neglected. The ligament releases had negligible effect on the tibiofemoral alignment. As ligament release can be considered as a change of the ligament stiffness without modification of the ligament length, the medial and lateral knee gaps, and consequently the tibiofemoral alignment, are not affected. This is in contrast to the gap-based approach, where the contact forces are kept constant with a distraction device, such as in the studies reported by Krackow and Mihalko (1999), Mihalko et al. (2003) or Baldini et al. (2004). In these cases, diminishing the ligament stiffness with a ligament release alters the tibiofemoral gap due to constraints on the force amplitude. Both methods, force-based and 
gap-based, are conceptually different. While the former measures directly the contact forces responsible for the component wear, the latter is limited by the complex force-displacement relationship of ligaments, which moreover is not necessarily identical on the medial and lateral side. Finally, the effect of ligament releases seems to vary along knee flexion. A different arrangement of the ligament fibers in the different positions could explain this variation. This again highlights the complexity of ligament balancing and the importance of an intraoperative force monitoring.

Overall, the device implantation was relatively fast and easy. Only the selection and insertion of spacers were time consuming. To avoid a significant increase of operating time, ergonomics should be improved by developing mechanically adjustable spacers for clinical routine use.

In conclusion, the device worked correctly under realistic conditions of use. In addition to the accurate imbalance assessment based on the measurement of forces and moments, important clinical advantages, such as the possibility to keep the patella in its anatomical position during the measurement or the real-time force monitoring during the delicate phase of ligament release, were demonstrated. The proposed device has thus shown potential to improve the ligament balancing procedure and the survivorship of TKA.

\section{Acknowledgements}

The authors would like to thank the Swiss National Science Foundation for the financial support through the National Center for Competence in Research CO-ME (Computer Aided and Image Guided Medical Interventions).

\section{References}

Attfield, S.F., Warren-Forward, M., Wilton, T., Sambatakakis, A., 1994. Measurement of soft tissue imbalance in total knee arthroplasty using electronic instrumentation. Medical Engineering and Physics 16, 501-505.

Baldini, A., Scuderi, G.R., Aglietti, P., Chalnick, D., Insall, J.N., 2004. Flexion-extension gap changes during total knee arthroplasty. The Journal of Knee Surgery 17 (2), 69-72.

Crottet, D., Maeder, T., Fritschy, D., Bleuler, H., Nolte, L.P., Pappas, I.P., 2005. Development of a force amplitude- and locationsensing device designed to improve the ligament balancing procedure in TKA. IEEE Transactions on Biomedical Engineering 52 (9), 1609-1611.

Decking, R., Markmann, Y., Fuchs, J., Puhl, W., Scharf, H.P., 2005. Leg axis after computer-navigated total knee arthroplasty. The Journal of Arthroplasty 20 (3), 282-288.

Dürselen, L., Claes, L., Kiefer, H., 1995. The influence of muscle forces and external loads on cruciate ligament strain. The American Journal of Sports Medicine 19, 381-383.

Fehring, T.K., Valadie, A.L., 1994. Knee instability after total knee arthoplasty. Clinical Orthopaedics and Related Research 299, $157-162$.

Haaker, R.G., Stockheim, M., Kamp, M., Proff, G., Breitenfelder, J., Ottersbach, A., 2005. Computer-assisted navigation increases precision of component placement in total knee arthroplasty. Clinical Ortho- paedics and Related Research 433, 152-159.

Jenny, J.-Y., Boeri, C., 2001. Computer-assisted implantation of total knee prostheses: a case-control comparative study with classical instrumentation. Computer Aided Surgery 6, 217-220.

Krackow, K.A., Mihalko, W.M., 1999. The effect of medial release on flexion and extension gaps in cadaveric knees. The American Journal of Knee Surgery 12, 222-228.

Lüring, C., Hüfner, T., Kendoff, D., Perlick, L., Bäthis, H., Grifka, J., 2005. Influence of everted and subluxed patella in ligament balancing. In: Proceedings of the Fifth Annual Meeting of CAOS-International, Helsinki, Finland.

Mihalko, W.M., Whiteside, L.A., Krackow, K.A., 2003. Comparison of ligament-balancing techniques during total knee arthroplasty. The Journal of Bone and Joint Surgery 85-A (Suppl. 4), 132-135.

Moreland, J.R., 1988. Mechanisms of failure in total knee arthroplasty. Clinical Orthopaedics and Related Research 226, 49-64.

Morris, B.A., D’Lima, D.D., Slamin, J., Kovacevic, N., Arms, S.W., Townsend, C.P., Colwell Jr., C.W., 2001. E-Knee: evolution of the electronic knee prosthesis. The Journal of Bone and Joint Surgery 83- A, 62-66.

Stulberg, S.D., 2003. How accurate is current TKR instrumentation. Clinical Orthopaedics and Related Research 416, $177-184$.

Takahashi, T., Wada, Y., Yamamoto, H., 1997. Soft-tissue balancing with pressure distribution during total knee arthroplasty. The Journal of Bone and Joint Surgery 79 (2-B), 235-239.

Wallace, A.L., Harris, M.L., Walsch, W.R., Bruce, W.J.M., 1998. Intraoperative assessment of tibiofemoral contact stresses in total knee arthroplasty. The Journal of Arthroplasty 13 (8), 923-927.

Winemaker, M.J., 2002. Perfect balance in total knee arthroplasty: the elusive compromise. The Journal of Arthroplasty 17 (1), $2-$ 10 . 